\title{
High temperature RF SQUIDs for biomedical applications
}

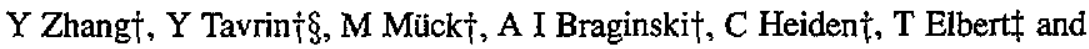 \\ $S$ Hampsont \\ † Forschungszentrum J"lich, Institut für Schicht- and Ionentechnik (ISI), D-5170 Jülich, \\ Germany \\ ‡ Westfälische Wilhelms-Universität, Institut für Experimentelle Audiologie. D-4400 Müinster, \\ Germany
}

Received 12 June 1992

\begin{abstract}
We have been investigating the feasibility of radio-frequency RF, low-noise superconducting quantum interference device (SQuID) magnetometers and gradiometers operating in liquid nitrogen at $77 \mathrm{~K}$. Using flux-focusing structures fabricated from epitaxial $\mathrm{YBa}_{2} \mathrm{Cu}_{3} \mathrm{O}_{7-x}$ films, we have attained a magnetic field resolution for a magnetometer of better than $200 \mathrm{fr}$ $\mathrm{Hz}^{-1 / 2}$ at less than $1 \mathrm{~Hz}$, i.e. over the low signal-frequency range important for biomedical diagnostics. At $77 \mathrm{~K}$, this magnetometer recorded diagnostically useful heart signals, voluntary eye-blink signals, and also the first evoked response of a human brain. These and similar results were obtained in a magnetically shielded room. We were also able to record heart signals in the absence of any shielding when using a first-order gradiometer. An improvement in the magnetic field resolution of our magnetometers and gradiometers by, at least, another onder of magnitude is possible and probable.
\end{abstract}

High transition temperature $\left(T_{c}\right)$ superconductors (HTS) hold the promise of a significant simplification and cost reduction of medical diagnostic equipment based on the use of superconducting quantum interference devices (SQUIDs). In the future, SQUDD-based measurements may replace much of today's routine electrophysiology. The driving force behind such a development originates from the simplicity and elegance of biomagnetic measurement; no electrodes are needed, the measuring device does not even touch the body. Without much preparation, data from many biomagnetic channels can be recorded without being affected by electrode drift, high electrode impedance or improper placement of electrodes. The often cited superiority of magnetic over electric data for source localization procedures may also play a role. Of course, low-cost high- $T_{\mathrm{c}}$ SQums having sufficient sensitivity need to be available first. Remarkable progress in the sensitivity of DC SQUIDs and $\mathrm{DC}$ SQUID magnetometers fabricated from thin-film $\mathrm{YBa}_{2} \mathrm{Cu}_{3} \mathrm{O}_{7-x}$ (YBCO) and the capacity to operate at temperatures around that of liquid nitrogen $(T=77 \mathrm{~K})$ has been reported recently by Miklich et al (1991). The basic feasibility of magnetocardiography was demonstrated by these authors, although their data were too noisy to be of diagnostic value. We have been investigating the possibility of attaining sensitivities sufficient for many biomagnetic applications of SQUIDs using much simpler high- $T_{\mathrm{c}}$ radio frequency (RF) SQUID magnetometers and gradiometers. We describe here a YBCO thin-film RF SQUID magnetometer having a high magnetic field resolution of $B_{\mathrm{n}}<200 \mathrm{fT} \mathrm{Hz}^{-1 / 2}$ at $77 \mathrm{~K}$, and demonstrate its potential for medical diagnostics. We have been able to record not only diagnostic-level magnetocardiograms but also, and for the first time, signal traces produced

$\S$ Permanent address: Institute of Low Temperature Physics, Ukrainian Academy of Sciences, Kharkov, CIS. 
by voluntary eye blinking and by acoustically evoked brain response. Biomagnetic signals encompass a large amplitude range, with those due to magnetized lung contaminants being strongest, up to $10^{3} \mathrm{pT}$. Abdominal currents generate fields about twice the intensity of the $\mathrm{R}$-wave of the cardiogram which is, at $5 \times 10^{4} \mathrm{fT}$, still about a thousand times stronger than the evoked activity of the cerebral cortex. The cardiogram fine structure can be at least an order of magnitude weaker than the $\mathrm{R}$-wave. The relevant signal-frequency range is between 0.3 and $60-80 \mathrm{~Hz}$, approximately. The SQUID device sensitivity must be adequate in that range. With a signal-to-noise ratio of 10 , a minimum sensitivity of $500 \mathrm{fT}$ is thus desired in a bandwidth of say $70 \mathrm{~Hz}$. This corresponds to a magnetic field resolution of $B_{\mathrm{n}} \approx 60 \mathrm{fT} \mathrm{Hz}-1 / 2$, and better resolutions would still be useful. A much better resolution, up to $B_{\mathrm{n}} \approx 3-5 \mathrm{fT} \mathrm{Hz}^{-1 / 2}$ may be required for clinical magnetoencephalography. The SQUID sensitivity is limited by the flux noise. For a RF SQUID, the intrinsic flux white noise spectral density is (Kurkijärvi 1973)

$$
S_{\Phi} \approx \frac{\left(L_{\mathrm{s}} I_{\mathrm{c}}\right)^{2}}{2 \pi f_{\mathrm{T}}}\left(\frac{2 \pi k_{\mathrm{B}} T}{l_{\mathrm{c}} \Phi_{0}}\right)^{4 / 3}
$$

where $L_{\mathrm{s}}$ is the SQUID inductance, $I_{\mathrm{c}}$ the critical current of the Josephson junction, $f_{\mathrm{T}}$ the RF SQUID tank frequency and $\Phi_{0}$ the flux quantum. Therefore, it is clear that to obtain a low $S_{\Phi}$, one has to use a high $f_{T}$ and a low $L_{\mathrm{s}}$. However, for optimum RF SQUID operation, the requirement

$$
\beta_{\mathrm{L}}=2 \pi I_{\mathrm{c}} L_{\mathrm{s}} / \Phi_{0} \approx 1
$$

should also be met. The minimum value of $L_{\mathrm{s}}$ must be large enough for a good coupling to the tank circuit and the signal source, e.g. the fiux transformer of a magnetometer. When (2) is optimized, the $I_{c}$ value is also fixed. That leaves $f_{\mathrm{T}}$ as the practical parameter which can be increased for noise reduction. While conventional RF SQUIDS usually operate with $f_{\mathrm{T}}=$ 10-20 MHz, we have been using values of $f_{\mathrm{T}}$ up to an order of magnitude higher (Zhang et al 1992). At the low signal frequencies of interest here, however, the SQuid sensitivity can be limited not by white noise but by the $1 / f$ or excess noise in the Josephson junction and SQUID structure. While that type of noise is generally high in high- $T_{\mathrm{c}}$ SQUIDs, we have shown that in manufacturable. $\mathrm{YBa}_{2} \mathrm{Cu}_{3} \mathrm{O}_{7-x}$ (YBCO) thin-film step-edge grain-boundary junctions it can, in fact, be relatively low (Herrmann et al 1991). This low noise level of step-edge junctions was also confirmed in DC SQUIDs (Friedl et al 1992). Furthermore, we have shown (Zhang et al 1992) that the $1 / f$-type of step-edge junction noise also scales with $f_{\mathrm{T}}$, at least in the flux-locked mode of operation.

For a simple demonstration of a sensitive YBCO thin-film RF SQUID we used large, $6 \times 6$ and $8 \times 8 \mathrm{~mm}^{2}$ flux-focusing washer structures (figure 1) with a double step-edge junction. SQUIDs with various $L_{\mathrm{s}}$ values, i.e. various hole sizes, have been fabricated and tested. Details of the washer and junction construction can be found in Zhang et al (1992).

Flux noise measurements were performed in liquid nitrogen, in a mumetal-shielded dewar using $f_{T}=150 \mathrm{MHz}$ and the flux-locked regime. A measured flux noise spectrum is shown in figure 2 for a $8 \times 8 \mathrm{~mm}^{2}$ washer with $L_{\mathrm{s}}=190 \mathrm{pH}$ and $\beta_{\mathrm{L}} \approx 1$. The summary of the SQUID chip parameters measured and calculated (Ketchen et al 1985) at this $L_{\mathrm{s}}$ value is included in table 1 . Noise levels recorded at two other $L_{\mathrm{s}}$ values are also indicated in figure 2. The lower value, $L_{\mathrm{s}}=25 \mathrm{pH}$, resulted in a $S_{\Phi}^{1 / 2}$ of only $3.5 \times 10^{-5} \Phi_{0} \mathrm{~Hz}^{1 / 2}$ down to below $0.3 \mathrm{~Hz}$, but coupling to the tank circuit was inadequate. The right-hand ordinate in figure 2 shows the magnetic field resolution at $L_{\mathrm{s}}=190 \mathrm{pH}$. It can be seen that the 


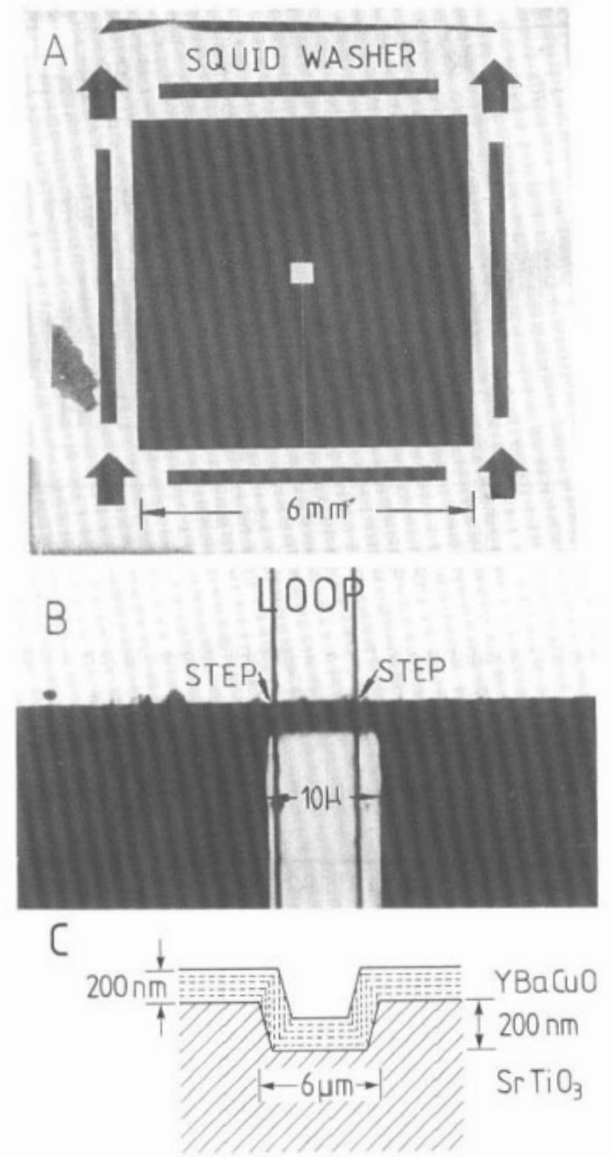

Figure 1. (a) A photograph of a $6 \times 6 \mathrm{~mm}^{2}$ YBCO RF SQUID washer with a $400 \times 400 \mu \mathrm{m}^{2}$ hole. (b) A magnified segment of the washer showing the $2 \mu \mathrm{m}$ wide and $10 \mu \mathrm{m}$ long mictobridge with two step-edge junctions created by a locak, $200 \mathrm{~nm}$ deep pit in the $\mathrm{SrTiO}_{3}$ substrate. (c) A schematic cross section of two junctions.

resolution of the $8 \times 8 \mathrm{~mm}^{2}$ SQUID is slightly better than $B_{\mathrm{n}}=200 \mathrm{fT} \mathrm{Hz}^{-1 / 2}$ down to 1 $\mathrm{Hz}$ and about $700 \mathrm{fT} \mathrm{Hz} z^{-1 / 2}$ at $0.1 \mathrm{~Hz}$. We show below that this sensitivity level is already sufficient to record real-time magnetocardiograms and signal-averaged encephalograms in a clinical magnetically shielded room. However, we point out that a better coupling to a smaller $L_{\mathrm{s}}$ can be obtained using a YBCO planar flux transformer, as shown for example by Lee et al (1991). Using their value of magnetic field gain of over 125 for an input coil with a $50 \times 50 \mu \mathrm{m}^{2}$ hole we can expect for our present sQu1D with $L_{\mathrm{s}}=190 \mathrm{pH}, B_{\mathrm{n}}=30 \mathrm{fT}$ $\mathrm{Hz}^{-1 / 2}$ at $77 \mathrm{~K}$ and above $1 \mathrm{~Hz}$. At very low signal frequencies, still better field resolutions could be obtained for lower values, $L_{\mathrm{s}}=50$ to $100 \mathrm{pH}$. Flux transformers with still higher field gains are also feasible.

In the shielded room, we measured the biomagnetic signal using our in-house designed $150 \mathrm{MHz}$ SQUID electronics with a bandwidth of $30 \mathrm{~Hz}$. An example of a magnetocardiographic trace of a male subject is shown in figures $3(a)$ and $(b)$. It was obtained at an arbitrary location of the SQU1D sensor positioned near the heart, just above 


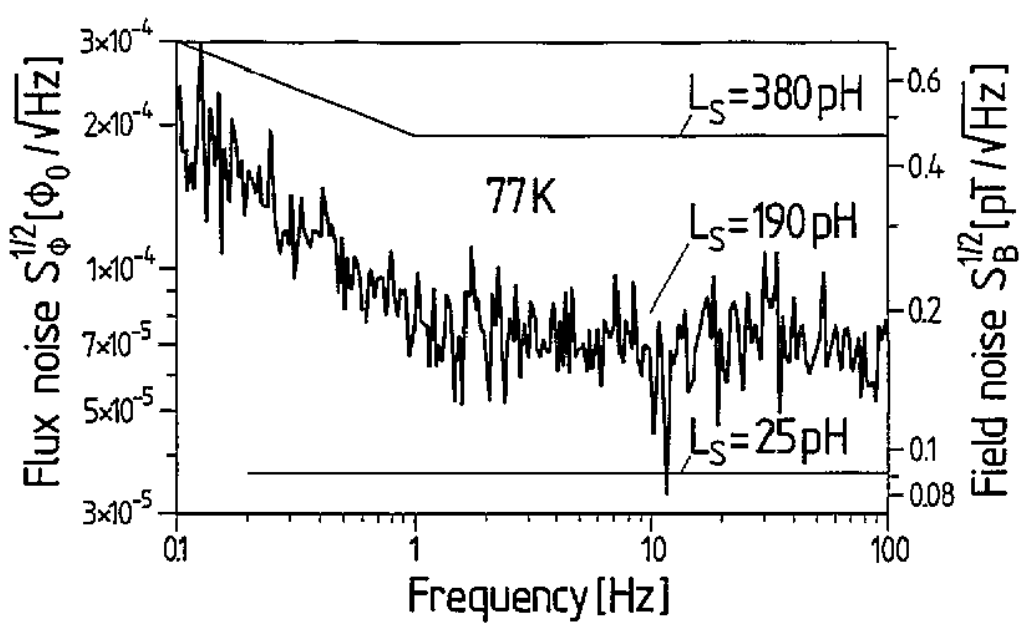

Figure 2. Noise spectrum of a washer with $L_{\mathrm{s}} \approx 190 \mathrm{pH}$. Noise levels for two other $L_{\mathrm{s}}$ values are indicated.

Table 1. Measured at $77 \mathrm{~K}$ and calculated data of RF SQuiDs with two different inductances, $\beta_{\mathrm{L}} \approx 1, \mathrm{f}_{\tau}=150 \mathrm{MHz}$.

\begin{tabular}{lll}
\hline Inductance, $L_{S}, \mathrm{pH}$ & 190 & 380 \\
Hole area, $d \times d, \mu \mathrm{m}^{2}$ & $150 \times 150$ & $300 \times 300$ \\
Washer area, $l \times l, \mathrm{~mm}^{2}$ & $8 \times 8$ & $6 \times 6$ \\
Transfer function, $\mathrm{d} V / \mathrm{d} \Phi, \mu \mathrm{V} \Phi_{0}^{-1}$ & 80 & 30 \\
Noise at $1 \mathrm{~Hz}, S_{\phi}^{1 / 2}, \Phi_{0} \mathrm{~Hz}^{-1 / 2}$ & $5 \times 10^{-29}$ & $2.3 \times 10^{-28}$ \\
Energy resolution at $1 \mathrm{~Hz}, \epsilon, \mathrm{J} \mathrm{Hz}{ }^{-1}$ & $5 \times 10^{-29}$ & $2.3 \times 10^{-28}$ \\
Magnetic field gain & & \\
$\quad$ Calculated (Ketchen et al 1985) & 59 & 22 \\
$\quad$ Measured & 38 & 14 \\
Field/flux transformation coefficent, $\mathrm{nT} \Phi_{0}^{-1}$ & 2.4 & 1.7 \\
Field resolution at $1 \mathrm{~Hz}, B_{\mathrm{n}}, \mathrm{fT} \mathrm{Hz}$ & $17 / 2$ & 360 \\
Device in which sQuid wras used & Magnetometer & Gradiometer \\
\hline
\end{tabular}

a Ratio of effective to geometrical loop area.

the subject's thorax. A comparison with reference traces obtained using a liquid-heliumcooled 37-channel clinical gradiometer (MAGNES, BTI) showed that our (strongly location dependent) traces are already of diagnostic quality, although obtained in the real-time mode, without any signal averaging and filtering. A magnetic signal of voluntary eye blinks obtained in the same mode is shown in figure 4. We verified that the ringing seen in this curve, and perhaps also in figure 3 , was due to AC coupling to our digital oscilloscope.

Finally, figure 5 shows the evoked response of a human brain triggered by an acoustic signal. A series of $1 \mathrm{KHz}, 60 \mathrm{~dB}$ tones, each $0.5 \mathrm{~s}$ in duration, was presented to the subject's left ear. The interval between two consecutive tones was set at $4 \mathrm{~s}$. The SQUID was placed over the right temporal region, near the posterior maximum of the M100, 15$20 \mathrm{~mm}$ above the subject's head. In this case, the magnetic activity was averaged over 80 trials, free of large amplitude artifacts, using the MAGNES system electronics. The signal is shown without and with low-pass filtering. Inspection of the averaged and filtered 

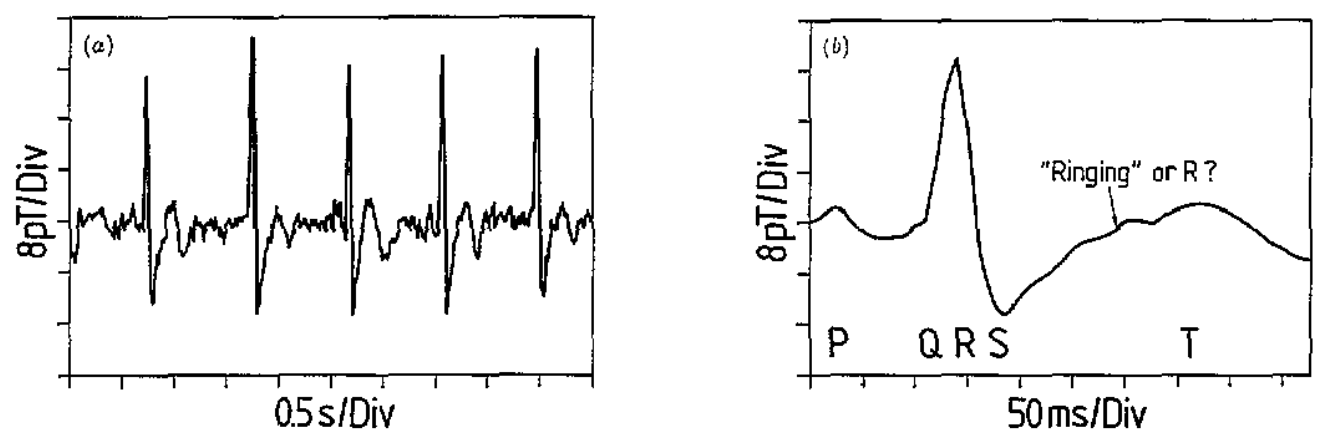

Figure 3. (a) Real-time magnetic signal of the heart obtained using our thin-film HTS magnetometer in a shielded room. (b) An expanded short segment of trace (a). The P-wave, the QRS-complex and the T-wave can be easily identified even in the raw trace $(b)$.

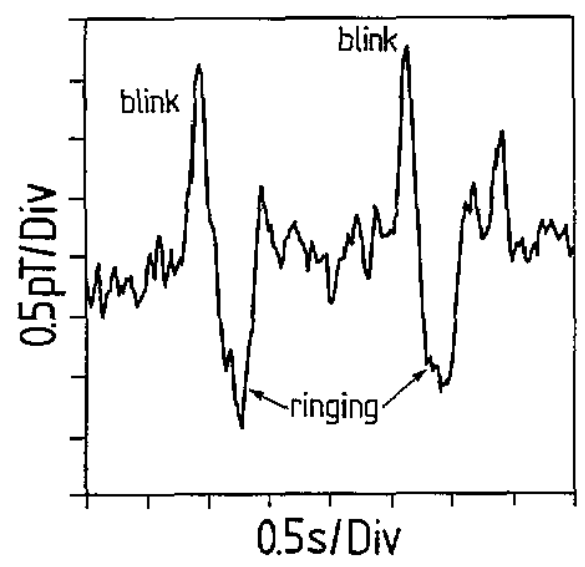

Figure 4. The subject was asked to perform several consecutive voluntary eye blinks. The real-time signal was recorded by placing our magnetometer at a position near the outer canthus of the left eye.

waveform reveals the well-known components M50, M100 and M200.

While further improvements of $B_{\mathrm{n}}$ are certainly required for clinical magnetocardiography and magnetoencephalography, figures 3-5 illustrate, we believe, the immediate potential of HTS RF SQUIDs for medical diagnostics. We also believe that our brain activity data are the first of their kind obtained at $77 \mathrm{~K}$ using an HTS SQUID.

The impact of SQUID devices on diagnostic equipment would certainly be greater if one could dispense with expensive shielded room environments. This will require using higher-order HTS gradiometers. We demonstrated the basic feasibility of this approach by constructing a HTS thin-film first-order electronic gradiometer which is described separately (Tavrin et al 1992). In-phase signals from two SQUIDs which were separated by a vertical base distance of $60 \mathrm{~mm}$ were substracted and filtered. In this case, two magnetometer chips 


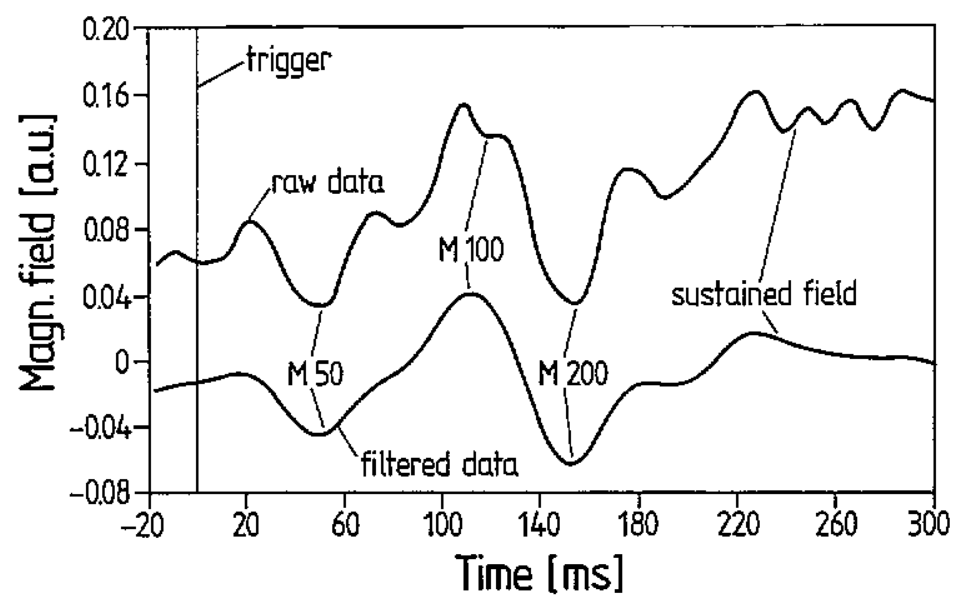

Figure 5. Averaged $(x 80)$ signal of acoustically evoked brain response recorded with our magnetometer. Unfiltered and filtered traces are shown. The description of the experiment is given in text.

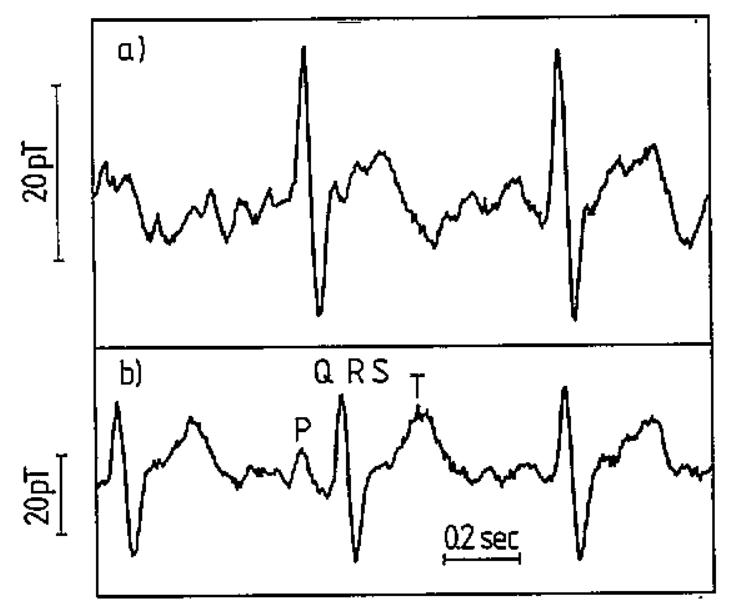

Figure 6. Real-time magnetic signal of one male subject's heart recorded (a) using the firstorder HTS RF SQUID gradiometer in the absence of any magnetic shielding and (b) using one channel of the gradiometer inside the shielded room (measurement done one day later).

with $L_{\mathrm{S}}=380 \mathrm{pH}$ have been used (chip data are included in table 1) and $f_{\mathrm{T}}=100 \mathrm{MHz}$. The common-mode rejection, i.e. the gradiometer balance, was about $10^{3}$ for distant $50 \mathrm{~Hz}$ signal sources.

The example of a measured real-time heart signal of the same male subject recorded in $(a)$ an electronic laboratory building environment, without any shielding, and $(b)$ in the shielded room using only one (magnetometer) channel is shown in figure 6 . In these measurements, the magnetic field resolution was $B_{\mathrm{n}}=500 \mathrm{fT} \mathrm{Hz}^{-1 / 2}$ (an improved version 
of the gradiometer described by Tavrin et al has $B_{\mathrm{n}}=280 \mathrm{fT} \mathrm{Hz}^{-1 / 2}$ ). The trace in figure $6(b)$ provides a reference base and shows that the structure of the gradiometer trace is not dominated by noise. The sensor's position over the heart was approximately the same in both measurements, which were performed at different times. The presented data are, we believe, the first of their kind obtained at $77 \mathrm{~K}$ in the absence of any shielding.

We are now planning to construct a second-order gradiometer configuration of magnetometer chips which will eventually be integrated with flux transformers. We believe that in such a case, and with signal averaging, the resulting portable device should have a sufficient reserve of sensitivity over the essential range of low signal frequencies and be entirely suitable for magnetocardiography in unshielded clinical environments.

We are grateful to Professor Dr med M Hoke, Medical Director of the Institute of Experimental Audiology for the support of this study and the encouragement offered to us. We also thank J Schubert and W Zander, KFA-ISI, who fabricated the YBCO films by pulsed laser deposition. Work at KFA was supported, in part, by the BMFT Consortium 'First Applications of HTS in Microelectronics'.

\section{References}

Friedl G, Vildic M, Rmheld M, Roas B, Uhl D, Bmmel F, Dalmaans G M, Hillenbrand B and Hoenig H E 1992 Low 1/f noise YBCO SQuid at $77 \mathrm{~K}$ Appl. Phys. Lett. at press

Hermann K, Zhang Y, M"ck M, Schubert J, Zander W and Braginski A I 1991 Characterization of YBCO step-edge Josephson junctions Supercond. Sci. Technol. 4 583-5

Ketchen M B, Gallagher W J, Kleinsasser A W, Murphy S and Clem J R 1985 De flux focuser SQUID'85-Superconducting Interference Devices and their Applications ed $\mathrm{H} D$ Hahlbohm and $\mathrm{H}$ Lübbig (Berlin: de Gruyter) pp 865-71

Kurkijärvi J 1973 Noise in the superconducting quantum flux detector $J$. Appl. Phys. 44 3729-33

Miklich A H, Kingston J J, Wellstood F C, Clarke J, Colclough M S, Char K and Zaharchuk G 1991 Thin film YBCO magnetometer Nature 352 482-3

Lee L P, Char K, Colclough M S and Zaharchuk G 1991 Monolithic 77 K DC SQuid magnetometer Appl. Phys. Lett. 59 3051-3

Tavrin Y, Zhang Y, Mueck M, Braginski A I and Heiden C 1992 A YBCO thin film sQuD gradiometer for biomagnetic measurements Appl. Phys. Lett. submitted

Zhang Y, M"ck M, Herrmann K, Schubert J, Zander W, Braginski A I and Heiden C 1992 low-noise YBCO RF SQuid magnetometer Appl. Phys. Lett. 60 645-7 